Corona-Krise

\section{Bildung und Soziale Marktwirtschaft}

Die Corona-Krise stellt die Gesellschaftsordnung vor noch nie dagewesene Herausforderungen. So haben die Pandemie und der damit einhergehende Lockdown eine weltweite Wirtschaftskrise ausgelöst, deren Folgen jene der Wirtschafts- und Finanzkrise 2008/2009 bei weitem übertreffen werden. Besonders bedenklich ist, dass die Corona-Krise zu einem deutlichen Anstieg von Ungleichheit führt. So besteht etwa für Arbeitnehmer*innen mit geringerem Bildungsstand oder Einkommen seltener die Möglichkeit, von zu Hause aus zu arbeiten. Gleichzeitig sind diese Arbeitnehmer*innen besonders stark von Kurzarbeit und Arbeitslosigkeit betroffen. Im Bildungsbereich zeigt sich, dass vor allem leistungsschwache Schüler*innen aufgrund der coronabedingten Schulausfälle besonders große Lerneinbußen verzeichnen, was die ohnehin schon hohe Bildungsungleichheit in Deutschland mittelfristig weiter verschärft.

Vor diesem Hintergrund stellt sich die Frage, wie die Soziale Marktwirtschaft auch in Krisenzeiten ihr zentrales Versprechen einlösen kann, alle Menschen zu eigenverantwortlicher Teilhabe am Markt und an der Gesellschaft zu befähigen. Die Antwort lautet: Bildung - und zwar eine Bildungspolitik, die das Ziel hat, gleich gute Startbedingungen für alle zu schaffen. Sie ist der Schlüssel, um die nötige Transformation zu bewerkstelligen. Die Forderung, die Soziale Marktwirtschaft stärker auf die Herstellung von gleichen Bildungschancen auszurichten, um den Herausforderungen unserer Zeit gerecht zu werden, ist nicht neu. Sie wurde bereits Anfang 2019 vom Wissenschaftlichen Beirat beim Bundeswirtschaftsministerium in der Stellungnahme „Bildungsgerechtigkeit als Kernelement der Sozialen Marktwirtschaft" betont. Neu ist jedoch, dass die Corona-Krise die soziale Lage nochmals deutlich verschärft.

Die sich daraus ergebenden Herausforderungen machen die Bedeutung einer neuen, verstärkt bildungsorientierten Sozialen Marktwirtschaft sehr deutlich. Denn Bildung befähigt Menschen nicht nur dazu, erfolgreich am wirtschaftlichen und gesellschaftlichen Leben teilzuhaben. Sie ist auch ein entscheidender Faktor dafür, sich an Änderungen des wirtschaftlichen Umfelds anpassen zu können und unter neuartigen Gegebenheiten, wie sie etwa seit der Corona-Krise vorliegen, erfolgreich zu sein. Umso bedenklicher ist es, dass in Deutschland bereits vor der Corona-Krise

(c) Der/die Autor(en) 2020. Open Access: Dieser Artikel wird unter der Creative Commons Namensnennung 4.0 International Lizenz (https:// creativecommons.org/licenses/by/4.0/deed.de) veröffentlicht.

Open Access wird durch die ZBW - Leibniz-Informationszentrum Wirtschaft gefördert. der Bildungserfolg von Kindern im internationalen Vergleich am stärksten vom familiären Hintergrund abhängt. So hinken etwa die Mathematikleistungen von 15-Jährigen aus sozial schlechtergestellten Familien jenen aus bessergestellten Familien um etwa vier Schuljahre hinterher. Gleichzeitig erhalten Kinder aus bessergestellten Familien 2,5-mal häufiger eine Gymnasialempfehlung als Kinder aus Arbeiterfamilien, selbst bei gleichen schulischen Leistungen. Möchte man verhindern, dass sich diese eklatante Ungleichheit in den Bildungschancen von Kindern durch die Corona-Krise weiter verschärft, ist eine verstärkte Ausrichtung auf Bildungsgerechtigkeit dringend angezeigt.

Die ökonomische Forschung weist darauf hin, dass es durchaus Politikmaßnahmen gibt, die zu mehr Bildungsgerechtigkeit führen könnten. Zum einen zeigt die Literatur zur frühkindlichen Bildung, dass sich Unterschiede in Fähigkeiten und Kompetenzen zwischen Individuen bereits sehr früh im Leben auftun und sich dann im Laufe der Jahre vergröBern. Eine hochqualitative öffentliche Kinderbetreuung ist damit ein erfolgversprechendes Instrument, um Chancengerechtigkeit herzustellen und mittelfristig Ungleichheit in der Gesellschaft zu verringern. In Deutschland würde dies eine engere Verzahnung von Familienpolitik - bislang für die frühkindliche Bildung verantwortlich - und Bildungspolitik erfordern. Neben einem deutlichen Ausbau der Krippenund Kitaplätze müssen diese Einrichtungen auch qualitativ so ausgelegt sein, dass sie Kinder aus benachteiligten Verhältnissen optimal auf ihre Schulzeit vorbereiten.

Zum anderen ergeben zahlreiche Studien, dass die frühe Aufteilung von Schulkindern auf verschiedene Schularten die Bildungsungleichheit verschärft. Deutschland zählt zu den Ländern, die diese Aufteilung besonders früh durchführen. Wenn Kinder nicht schon mit zehn Jahren, sondern erst deutlich später auf verschiedene Schularten aufgeteilt würden, würde dies vor allem die Chancen von Kindern aus benachteiligten Verhältnissen erhöhen. Dazu könnten auch gezielte Fördermaßnahmen beitragen. Das derzeit vorherrschende Gießkannenprinzip bei der Verteilung von finanziellen Mitteln im Bildungsbereich eignet sich nicht, um Bildungsungleichheit ernsthaft zu bekämpfen. Gezielte Maßnahmen für benachteiligte Gruppen, etwa in Form von Mentoring-Programmen, wären eine deutlich wirkungsvollere Mittelverwendung. Das Ziel von Bildungspolitik als zentralem Element der Sozialen Marktwirtschaft muss sein, dass jedes Kind sein volles Bildungspotenzial ausschöpfen kann. Die Corona-Krise und ihre Folgen machen bewusst, dass es an der Zeit ist, die deutsche Bildungspolitik an der Erreichung dieses Ziels zu messen.

Philipp Lergetporer, Larissa Zierow ifo Zentrum für Bildungsökonomik, ifo Institut München zierow@ifo.de 